\title{
Mechanical Thrombectomy and Thrombolysis with Stent Placement for Superior Mesenteric Artery Thrombosis in a COVID-19 Patient
}

\author{
Abdulmajeed Bin Dahmash ${ }^{1}$ Aljabriyah Alfutais ${ }^{2}$ \\ ${ }^{1}$ Vascular and Interventional Radiology Unit, Department of Medical \\ Imaging, King Abdulaziz Medical City \& King Abdullah Specialized \\ Children's Hospital, Ministry of National Guard-Health Affairs, \\ Riyadh, Saudi Arabia \\ 2 Vascular and Interventional Radiology Unit, Department of Medical \\ Imaging, King Abdullah Bin Abdulaziz University Hospital, Princess \\ Nourah Bint Abdulrahman University, Riyadh, Saudi Arabia
}

Avicenna J Med 2022;12:38-40.

\author{
Shaker Alshehri ${ }^{1}$ Omar Bashir ${ }^{1}$
}

Address for correspondence Omar Bashir, MBBS, FRCR, EBIR, Vascular and Interventional Radiology Section, Department of Medical Imaging, King Abdulaziz Medical City, Ministry of National GuardHealth Affairs, Riyadh 22490-11426, Kingdom of Saudi Arabia (e-mail: drobashir@gmail.com).
COVID-19 primarily affects the respiratory system, from mild to severe symptoms such as acute respiratory distress syndrome. Owing to the global inflammatory response and endothelial damage, COVID-19 may predispose to coagulation disorders that can lead to thrombotic complications. ${ }^{1,2}$ Here, we present a case of superior mesenteric artery (SMA) thrombosis as a consequence of COVID-19, which was treated with an endovascular approach by mechanical thrombectomy with thrombolysis and stent placement in the SMA.

An 88-year-old patient with a history of diabetes, hypertension, and end-stage renal disease presented to the emergency department complaining of abdominal pain and vomiting. She was COVID-19 positive without fever or respiratory symptoms. Contrast-enhanced abdominal computed tomography (CT) scan demonstrated SMA thrombosis with diffusely reduced bowel enhancement ( $\sim$ Fig。 1). The patient was considered high risk for surgical intervention and was referred to interventional radiology (IR) for endovascular revascularization of the SMA. SMA angiography confirmed proximal thrombosis with preserved flow to jejunal branches and absent flow to colonic branches (-Fig. 2). Next, $4 \mathrm{mg}$ recombinant tissue plasminogen activator (rtPA) was used to lace the thrombus, and overnight thrombolysis was performed at a rate of $1 \mathrm{mg} / \mathrm{h}$ for 16 hours using a $5 \mathrm{Fr}, 7 \mathrm{~cm}$ length Cragg-McNamara infusion catheter (Medtronic, Minneapolis). Subsequent angiography demonstrated significant residual thrombus within the SMA. Using left brachial access with a $90 \mathrm{~cm} 6$-Fr sheath, successful thrombectomy was performed using CAT6 Indigo System (Penumbra, Alameda, CA, USA). Following this, a $7 \mathrm{~mm} \times 36 \mathrm{~mm}$ Valeo stent (Bard Inc., New Providence, NJ, USA) was deployed at the SMA origin to treat residual stenosis (-Fig. 3). The final angiogram demonstrated an excellent flow through the SMA and its main branches ( - Fig. 4). The patient developed left brachial artery vascular sheath-related dissection and thrombosis that required placement of a $5 \mathrm{~mm} \times 5 \mathrm{~cm}$ Viabahn stent graft (WL Gore, Flagstaff, Ariz, USA). The patient was discharged home after improving clinically and resuming oral intake. Two months later, the patient presented with bowel ischemia. However, a CT scan showed a patent celiac axis and SMA stent with occluded inferior mesenteric artery and thrombosis in one of the distal SMA branches. The patient passed away due to bowel ischemia complicated by sepsis and disseminated intravascular coagulation.

COVID-19 patients can develop severe coagulopathy even without respiratory symptoms. ${ }^{3}$ Previous cases in the literature with SMA thrombosis related to COVID-19 were treated surgically and one medically with a high mortality rate. ${ }^{4}$ This case demonstrates that arterial thrombotic events in the context of COVID-19 may be treated with endovascular techniques to obviate the need for surgical interventions. Additionally, it illustrates the severe thrombotic complications of COVID-19. published online

February 21, 2022
DOI https://doi.org/ 10.1055/s-0041-1740645. ISSN 2231-0770. (c) 2022. Syrian American Medical Society. All rights reserved. This is an open access article published by Thieme under the terms of the Creative Commons Attribution-NonDerivative-NonCommercial-License, permitting copying and reproduction so long as the original work is given appropriate credit. Contents may not be used for commercial purposes, or adapted, remixed, transformed or built upon. (https://creativecommons.org/ licenses/by-nc-nd/4.0/)

Thieme Medical and Scientific Publishers Pvt. Ltd., A-12, 2nd Floor, Sector 2, Noida-201301 UP, India 


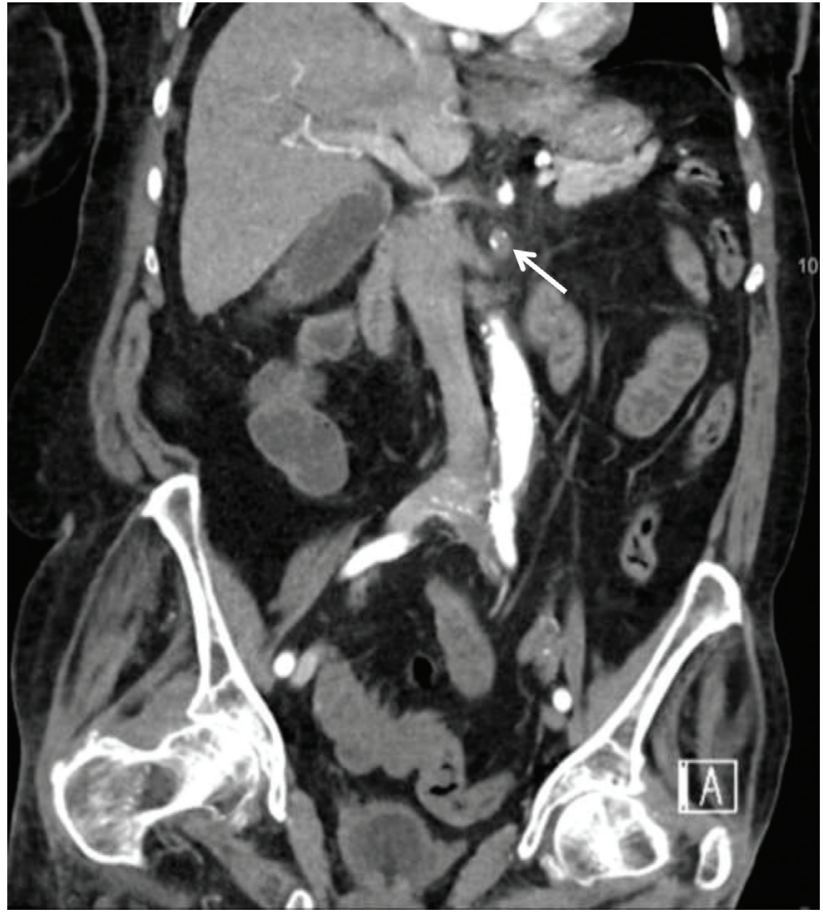

Fig. 1 Coronal CT image of the abdomen in arterial phase showing filling thrombosis of the superior mesenteric artery (arrow). CT, computed tomography.

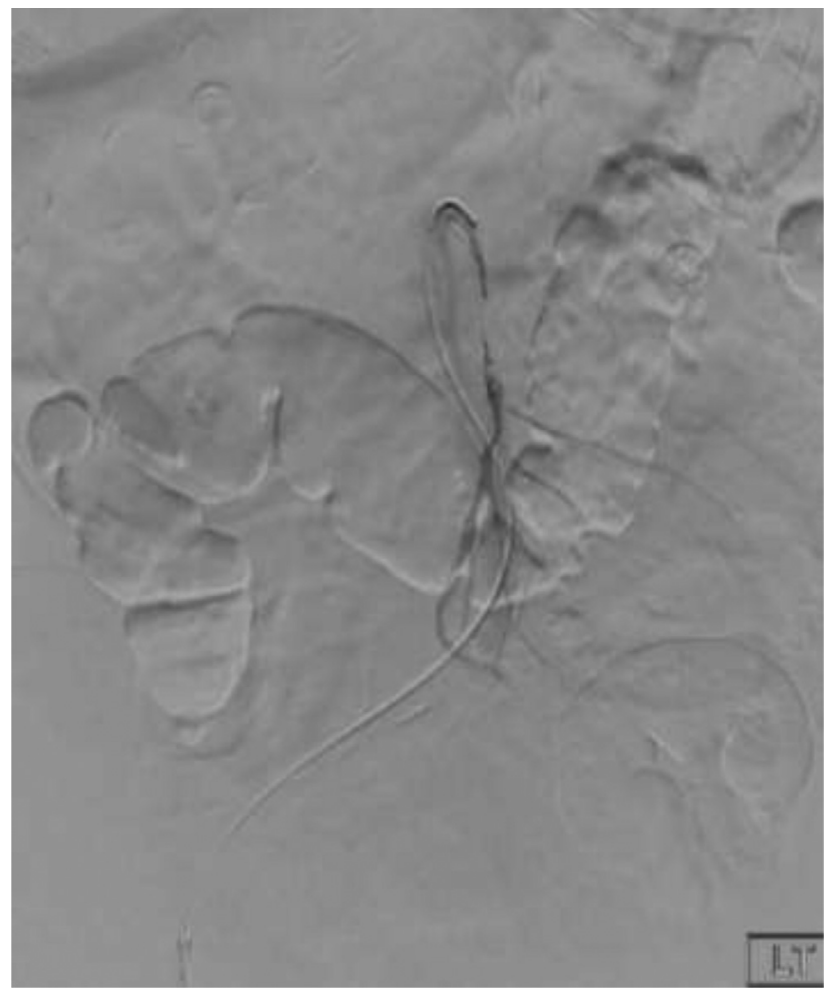

Fig. 2 SMA angiography showing the filling defect of the proximal part.

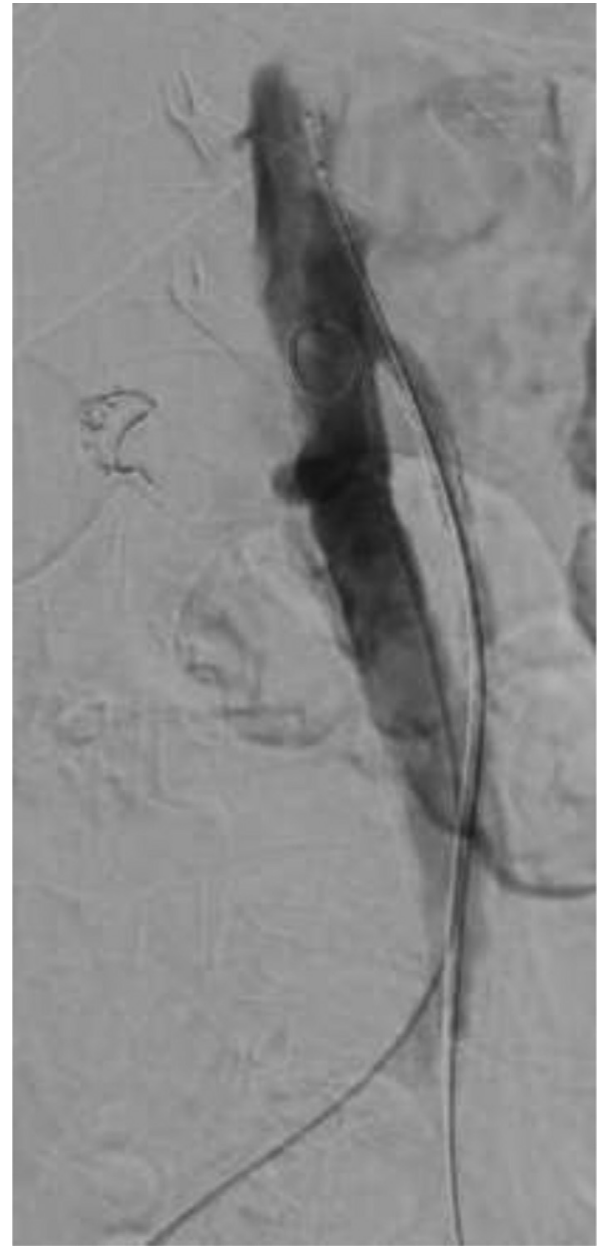

Fig. 3 SMA angiography post thrombectomy and stent placement.

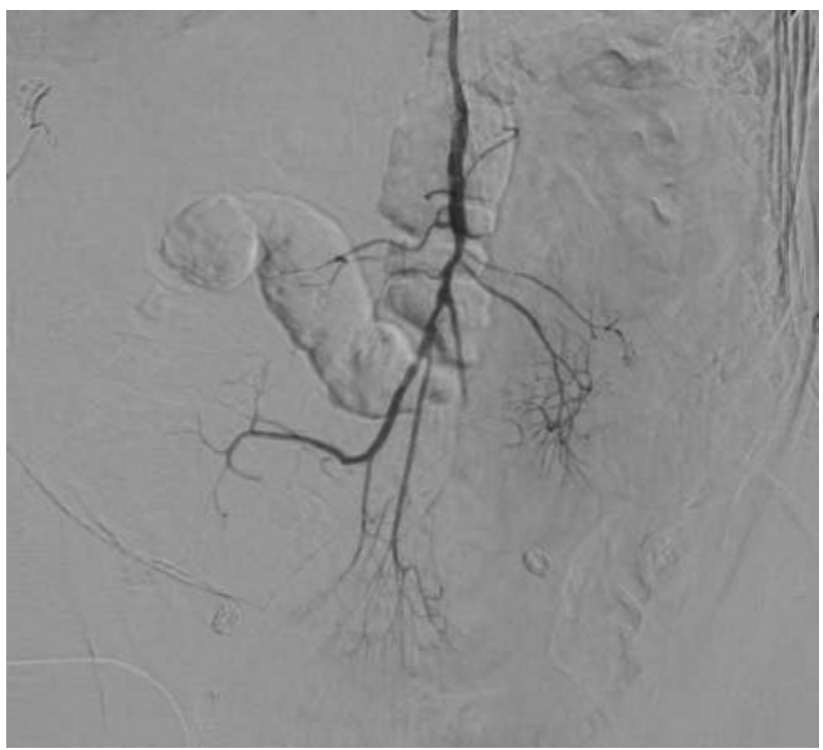

Fig. 4 Final angiogram showing improved flow in the superior mesenteric artery and its branches. 
Funding

None.

Conflict of Interest

None declared.

\section{References}

1 Zhang Y, Xiao M, Zhang S, et al. Coagulopathy and antiphospholipid antibodies in patients with Covid-19. N Engl J Med 2020;382 (17):e38. Doi: 10.1056/NEJMc2007575
2 Vulliamy P, Jacob S, Davenport RA. Acute aorto-iliac and mesenteric arterial thromboses as presenting features of COVID-19. Br J Haematol 2020;189(06):1053-1054. Doi: $10.1111 /$ bjh. 16760

3 Cheung S, Quiwa JC, Pillai A, Onwu C, Tharayil ZJ, Gupta R. Superior mesenteric artery thrombosis and acute intestinal ischemia as a consequence of COVID-19 infection. Am J Case Rep 2020;21: e925753. Doi: 10.12659/AJCR.925753

4 Amaravathi U, Balamurugan N, Muthu Pillai V, Ayyan SM. Superior mesenteric arterial and venous thrombosis in COVID-19.J Emerg Med 2021;60(05):e103-e107. Doi: 10.1016/j.jemermed.2020.12.016 